\title{
On-Chip Fabrication of Glass Sphere Laser
}

\author{
Tetsuo Kishi ${ }^{\text {a }}$, Tsutaru Kumagai and Tetsuji Yano \\ Department of Chemistry and Materials Science, Tokyo Institute of Technology, 152-8550 2-12-1 Ookayama, Meguro-ku, Tokyo, Japan
}

\begin{abstract}
Fabrication and application of glass spherical micro-cavity for lasing are reported. Surface-tension molding (StM) and localized-laser heating (LLH) techniques have been developed to fabricate glass super sphere, which is partially truncated spherical shape, and true spheres, respectively. Whispering gallery mode (WGM) resonances or laser oscillations from the spherical glasses were demonstrated. Super-spherical glasses possessed WGM resonances on its equatorial plane. The equatorial plane with high roundness $(>0.99)$ serves a high quality factor to lead laser oscillation. LLH technique enables us to fabricate true spheres on a transparent substrate. Tellurite glass spheres prepared by the LLH technique showed laser oscillation with few-mW-order thresholds by direct pumping. StM and LLH technique are very suitable for both preparation and utilization of glass spheres for optical micro-cavity.
\end{abstract}

\section{Introduction}

Micrometer-size glass spheres have received considerable attentions for many years mainly because of their versatility in micro-optical system for microlasers, microamplifiers, nonlinear optical devices, and chemical/bio sensors. However, it was difficult to fabricate microspheres having high sphericity and very smooth surface on a substrate, because the shape of molten glass droplet on substrate is determined by the wetting property of the substrate by the molten glass. We have developed a Surface-tension Molding (StM) and a localized-laser heating (LLH) technique for fabrication of micrometer-size spherical glass particles on a substrate ${ }^{1,2)}$. Their characteristics as microlasers were demonstrated.

\section{Surface tension Molding technique}

The surface-tension mold (StM) technique has been developed to fabricate micrometer-size super spherical glass particles, which are truncated spheres. The basic principle used in the fabrication of these spherical glasses is Young-Dupré formalism to control their shape. The crushed glass particles were heat-treated on glassy-carbon substrate, which is suitable for the formation of sphericalshaped molten glass droplets. Figure 1 shows scanning electron microscopy (SEM) image of the prepared super spherical glasses made of $\mathrm{Na}_{2} \mathrm{O}-\mathrm{CaO}-\mathrm{SiO}_{2}$ glass. This SEM images show that the fabricated glass particles have a flat plane resulting from their contact with the glassy carbon substrate and that the particles exhibit a truncate spherical shape (contact angle $\theta>90^{\circ}$ ).

\footnotetext{
a Corresponding author: tkishi@ceram.titech.ac.jp
}
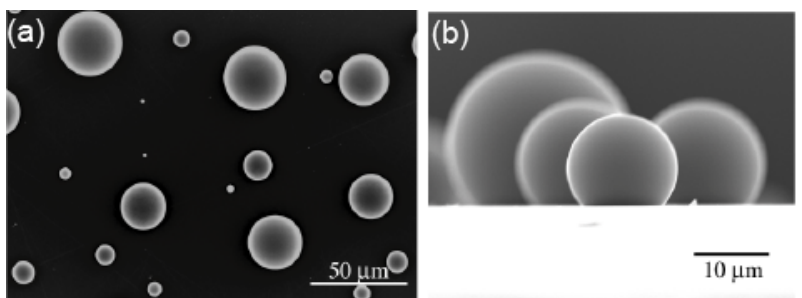

Figure 1. SEM images of glass truncated spheres with $\mathrm{Na}_{2} \mathrm{O}-\mathrm{CaO}-\mathrm{SiO}_{2}$. Top view (a) and side view (b) of truncated spheres prepared from crushed glass particles.

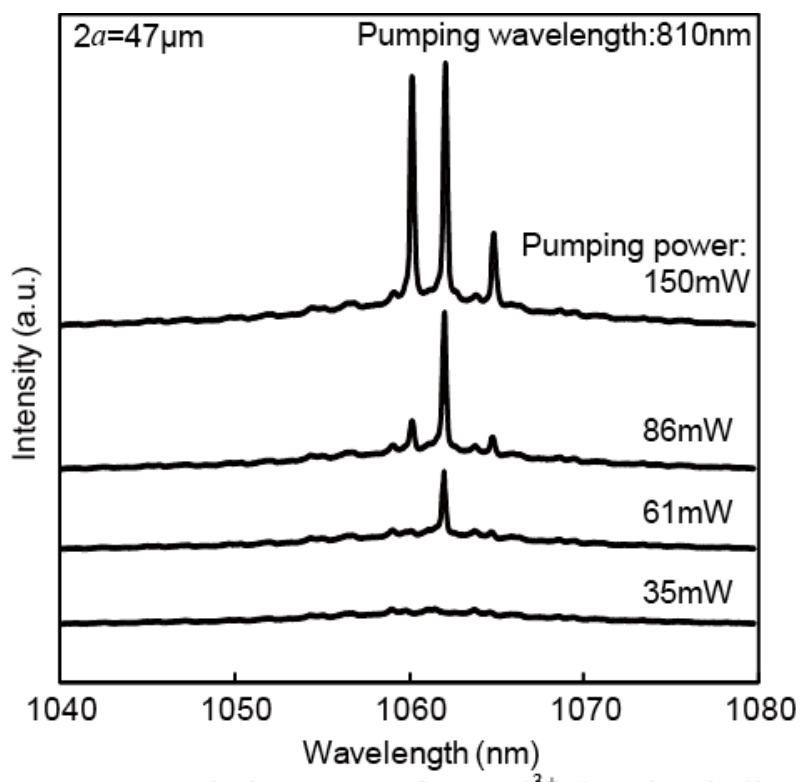

Figure 2. Emission spectra from $\mathrm{Nd}^{3+}$-doped soda-lime silicate glass truncated sphere prepared by StM technique.

Figure 2 shows the example of lasing spectrum of $\mathrm{Nd}^{3+}$-doped $\mathrm{Na}_{2} \mathrm{O}-\mathrm{CaO}-\mathrm{SiO}_{2}$ glass truncated sphere with

This is an Open Access article distributed under the terms of the Creative Commons Attribution License 4.0, which permits unrestricted use, distribution, and reproduction in any medium, provided the original work is properly cited. 
a diameter of $47 \mu \mathrm{m}$ excited by an $810-\mathrm{nm}$ band of ${ }^{4} \mathbf{I}_{9 / 2^{-}}$ ${ }^{4} \mathrm{~F}_{5 / 2}$ transition ${ }^{3)}$. Lasing lines (WGMs) due to the ${ }^{4} \mathrm{~F}_{3 / 2}{ }^{-}$ ${ }^{4} I_{11 / 2}$ transition were observed from the sphere. Broad natural luminescence is negligibly small compared to the lasing lines. The lasing threshold of this truncated spherical optical cavity at the $1.06-\mu \mathrm{m}$ line was $40 \mathrm{~mW}$. Deviation of the equatorial line from a true circle in the case of the truncated spheres fabricated by the StM technique does not always favor a high quality factor.

\section{Localized laser heating technique}

The localized-laser heating (LLH) technique enables nonadhesion melting of glass on substrate ${ }^{2)}$. LLH involves the laser heating of a glass particle on a certain transparent substrate. The crushed glass powder with a particle diameter of 10-100 $\mu \mathrm{m}$ was dispersed onto silica glass substrate. A focused laser beam is used to irradiate each particle. When the glass particle is doped with elements that absorb the laser light, non-radiative decay raise the temperature of the glass particles. Operation of the laser at high power melts the glass particles on the transparent substrate. Figure 3(a) and 3(b) show the sample heat-treated on a silica glass substrate at $390{ }^{\circ} \mathrm{C}$ for $30 \mathrm{~min}$ in an oven and the sample fabricated on the same substrate by the LLH technique, respectively. The glass cullet prepared by the conventional heat treatment had a plano-convex-lens shape, and that of the LLH technique had an almost true spherical shape. This means that the wetting of molten glass on substrate was suppressed in the case of the LLH, and true spheres are obtained on a transparent substrate as if they were melted under low gravity.

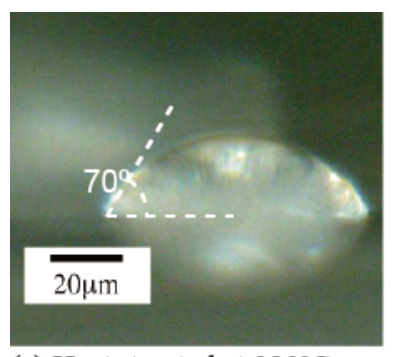

(a) Heat- treated at $390^{\circ} \mathrm{C}$.

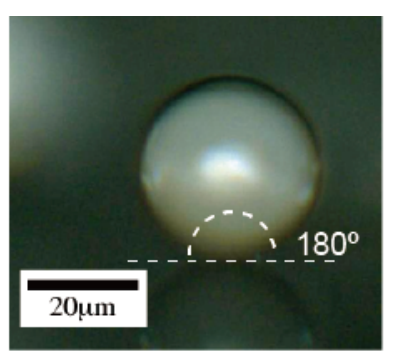

(b) Glass sphere fabricated by LLH technique
Figure 3. Side-view optical photographs of glass particles on silica glass substrate heat-treated by an oven (a), and fabricated by LLH technique.

Emission spectra from a Nd ${ }^{3+}$-doped $\mathrm{K}_{2} \mathrm{O}-\mathrm{WO}_{3}-\mathrm{TeO}_{2}$ glass microsphere with $29-\mu \mathrm{m}$ diameter fabricated by the LLH technique for various pumping intensities are shown in Figure 4. The pumping positions were the edge of the sphere, and the pumping wavelength was tuned to obtain the highest emission intensity: $803.5 \mathrm{~nm}$. The emission spectra shown in Fig. 4 include several sharp peaks and many broad weak peaks. The positions of peaks in the spectrum of the microsphere agree well with the theoretically estimated wavelength for a WGM resonator. The sharp peaks were attributed to laser emission because they had obvious thresholds $(1.3 \mathrm{~mW})$ as the excitation power increased. The $\mathrm{Q}$ values estimated from the full width at half maximum of the peaks below the thresholds were approximately $1 \times 10^{4}$ for the sphere, whose Q value was limited by the measurement system.

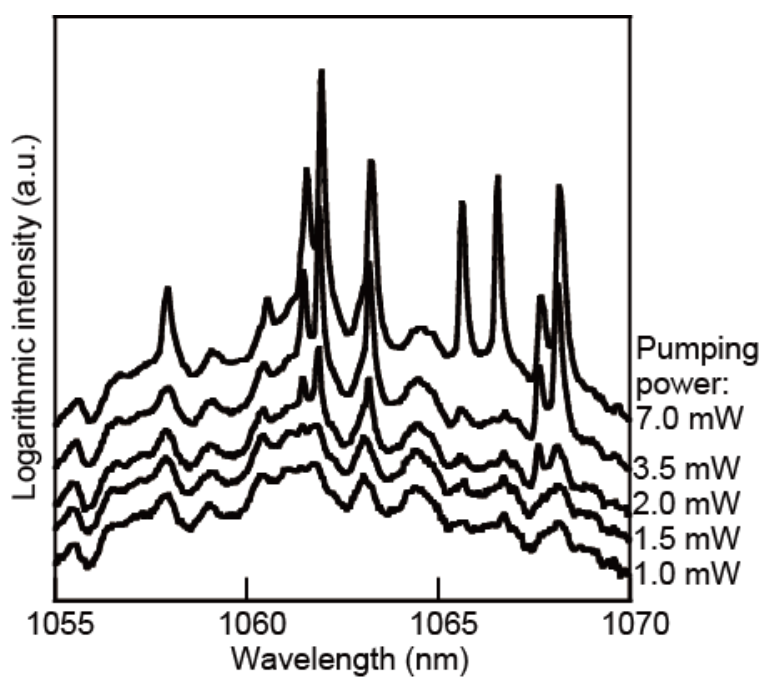

Figure 4. Emission spectra from a Nd ${ }^{3+}$-doped tellurite glass sphere $(2 \mathrm{a}=29 \mu \mathrm{m})$. Pumping wavelength is $803.5 \mathrm{~nm}$.

\section{Conclusion}

The spherical glass particles for microlaser were fabricated by surface-tension driven techniques (StM and LLH). The StM technique forms truncated spherical shapes by taking advantage of wetting property due to the Young-Dupré formalism. This simple process enables the fabrication of optical cavities of truncated spheres using its equatorial circle for WGM. The LLH technique enables the fabrication of glass spheres on a transparent substrate. This high-Q optical cavity exhibits a low threshold for lasing action. Characteristic glass properties enable these processes and enable the realization of high optical functionalities.

\section{References}

1. T. Kishi, S. Shibata and T. Yano, "Preparation of micrometer-size spherical glasses for optical resonator," Proceeding of XX International Congress of Glass, O-14-022-1-6 (2004) Kyoto.

2. T. Kishi, T. Kumagai, T. Yano, and S. Shibata, "Onchip fabrication of air-bubble-containing $\mathrm{Nd}^{3+}$-doped tellurite glass microsphere for laser emission," $A I P$ Advances 2, 042169-1-5 (2012).

3. T. Yano, T. Kishi, S. Shibata, T. Sato, A Inoki, unpublished data. 